\title{
Terminating Sequent Calculi for Two Intuitionistic Modal Logics
}

\author{
Rosalie Iemhoff *
}

January 4, 2020

\begin{abstract}
This paper presents sequent calculi in which proof search is terminating for two intuitionistic modal logics, the intuitionistic versions of the classical modal logics $\mathrm{K}$ and KD without a diamond operator. The calculi are extensions of the terminating sequent calculus G4ip for intuitionistic propositional logic that was discovered independently by Dyckhoff and Hudelmaier around 1990. It is shown by proof-theoretic means that these terminating calculi are equivalent to the cutfree extensions of G3ip that form some of the standard calculi for intuitionistic modal logics.
\end{abstract}

Keywords: intuitionistic modal logic, sequent calculus, termination

MSC: 03B05, 03B45, 03F03

\section{Introduction}

For several standard sequent calculi for classical propositional logic CPC, a corresponding calculus for intuitionistic propositional logic IPC can be obtained by restricting the right side of the sequents to one formula or, depending on the calculus, at most one formula. For the well-known calculus G3cp in (Troelstra and Schwichtenberg, 1996) this is no longer the case. This calculus does not contain structural rules, which are admissible in it, and restricting the right side of the sequents to exactly one formula does not result in a calculus for intuitionistic propositional logic, as some of the structural rules are no longer admissible. This defect can be remedied by slightly reformulating some of the rules. In this way a calculus G3ip for intuitionistic propositional logic without structural rules is obtained. But an important property of the classical system G3cp is lost. Namely, proof search in G3ip is no longer terminating.

The lack of termination makes G3ip unfit for certain proof-theoretic arguments about IPC, such as the proof that IPC has uniform interpolation by Pitts (1992). For his intricate proof Pitts uses a terminating calculus for IPC developed independently by Dyckhoff (1992) and Hudelmaier (1988, 1992, 1993)

\footnotetext{
*Utrecht University, Janskerkhof 13, 3512 BL Utrecht, the Netherlands, r.iemhoff@uu.nl.
} 
and, much earlier in a somewhat different form, by Vorob'ev $(1952,1970)$. This calculus, that, following Troelstra and Schwichtenberg (1996), is called G4ip in this paper, is the result of replacing the left implication rule in G3ip by four implication rules. Proof search in this calculus always terminates because in all rules in the calculus the premisses come before the conclusion in an order on sequents introduced in (Dyckhoff, 1992) and also used in this paper.

In this paper we extend these results to the intuitionistic versions of the classical modal logics $\mathrm{K}$ and KD without a diamond operator, thereby obtaining terminating cutfree sequent calculi for these logics. Both calculi are extensions of G4ip in which the structural rules are admissible. We prove them to be equivalent to the more standard calculi for these logics, namely calculi that are extensions of G3ip in which the structural rules are admissible.

These are modest results: one has to discover the right calculi, but once that has been achieved, the rest is straightforward. The reason for publishing these observations as a stand-alone paper is that terminating calculi potentially have many applications. For example, in a forthcoming paper (Iemhoff, 2018b) we establish that several intuitionistic modal logics have uniform interpolation, and these results use the terminating sequent calculi without structural rules developed in this paper. Whether these calculi will indeed find such applications elsewhere, only the future will tell.

\section{Logics and calculi}

The language $\mathcal{L}$ for intuitionistic modal propositional logic considered here contains a constant $\perp$, propositional variables or atoms $p, q, r, \ldots$, the modal operator $\square$ and the connectives $\neg, \wedge, \vee, \rightarrow$, where $\neg \varphi$ is defined as $\varphi \rightarrow \perp$. $\perp$ is by definition not an atom. $\mathcal{F}$ denotes the set of formulas in $\mathcal{L}$. Note that $\diamond$ does not occur in our language. For two multisets $\Gamma$ and $\Pi$, we denote by $\Gamma \cup \Pi$ the multiset that contains only formulas $\varphi$ that belong to $\Gamma$ or $\Pi$ and the number of occurrences of $\varphi$ in $\Gamma \cup \Pi$ is the sum of the occurrences of $\varphi$ in $\Gamma$ and in $\Pi$. For a (multi)set $\Gamma$ of formulas, $\square \Gamma$ denotes the (multi)set $\Gamma \cup\{\square \varphi \mid \varphi \in \Gamma\}$. $\bullet \varphi$ is short for $\varphi \wedge \square \varphi$, but if the expression occurs as an element of a sequent it stands for $\varphi, \square \varphi$, meaning that $(\Gamma, \bullet \varphi \Rightarrow \Delta)$ should be read as $(\Gamma, \square \varphi, \varphi \Rightarrow \Delta)$.

We only consider (single-conclusion) sequents, which are expressions $\Gamma \Rightarrow \Delta$, where $\Gamma$ and $\Delta$ are finite multisets of formulas and $\Delta$ contains at most one formula. Such sequents are interpreted as $I(\Gamma \Rightarrow \Delta)=(\bigwedge \Gamma \rightarrow \bigvee \Delta)$. We denote finite multisets by $\Gamma, \Pi, \Delta, \Sigma$. In a sequent, notation $\Pi, \Gamma$ is short for $\Gamma \cup \Pi$. In writing an expression $\Gamma \Rightarrow \Delta$ it is understood that $\Delta$ contains at most one formula. We also define ( $a$ for antecedent, $s$ for succedent):

$$
(\Gamma \Rightarrow \Delta)^{a} \equiv_{d f} \Gamma \quad(\Gamma \Rightarrow \Delta)^{s} \equiv_{d f} \Delta .
$$

When sequents are used in the setting of formulas, we often write $S$ for $I(S)$, such as in $\vdash \bigvee_{i}\left(S_{i} \rightarrow S\right)$, which thus denotes $\vdash \bigvee_{i}\left(I\left(S_{i}\right) \rightarrow I(S)\right)$. 
Given a sequent calculus $\mathrm{G}$ and a sequent $S, \vdash_{\mathrm{G}} S$ denotes that $S$ is derivable in $G$. The logic $L_{G}$ corresponding to $G$ is defined as

$$
\vdash_{\mathrm{L}_{\mathrm{G}}} \varphi \equiv_{d f} \vdash_{\mathrm{G}}(\Rightarrow \varphi) .
$$

The four main sequent calculi in this paper, G3iK $\square, G 4 i K_{\square}, G_{i K D_{\square}}, G 4 i K D_{\square}$, correspond to the two logics which are most commonly called $\mathrm{iK}_{\square}$ and $\mathrm{iKD}_{\square}$. In Section 7.1 the connection to intuitionistic modal logics in the literature is dicussed further. Since we wish not to include completeness proofs in this paper, we chose to define the logic of a calculus as above, and leave the proofs that these logics correspond to the ones in the literature to the reader.

We use an order on sequents based on the weight function $w(\cdot)$ on formulas from (Dyckhoff, 1992), which is inductively defined as: the weight of an atom and the constant $\perp$ is $1, w(\square \varphi)=w(\varphi)+1$, and $w(\varphi \circ \psi)=w(\varphi)+w(\psi)+i$, where $i=1$ in case $\circ \in\{\bigvee, \rightarrow\}$ and $i=2$ otherwise. We use the following ordering on sequents: $S_{0} \ll S_{1}$ if and only if $S_{0}^{a} \cup S_{0}^{s} \ll S_{1}^{a} \cup S_{1}^{s}$, where $\ll$ is the order on multisets determined by weight as in (Dershowitz and Manna, 1979) (where they in fact define $\gg$ ): for multisets $\Gamma, \Delta$ we have $\Delta \ll \Gamma$ if $\Delta$ is the result of replacing one or more formulas in $\Gamma$ by zero or more formulas of lower weight.

\subsection{Intuitionistic modal logic}

The calculus G4ip in Figure 2.1 is the calculus independently discovered by Dyckhoff (1992) and Hudelmaier (1988, 1992, 1993), with different names for some of the rules. Recall that in this paper sequents are assumed to have at most one formula in the succedent, which is a slight but inessential deviation from the calculus by Dyckhoff and Hudelmaier, where sequents are assumed to have exactly one formula at the right. The calculus is terminating with respect to the above well-ordering $\ll$ on sequents. The calculus has no structural rules, but they are admissible in it, as is shown below.

The calculus G3ip, given in Figure 2.2, consists of the rules of G4ip where the four left implication rules are replaced by one implication rule, $L \rightarrow$. It is the propositional part of the calculus G3i from (Troelstra and Schwichtenberg, 1996). When one analyses the nontermination of G3ip, one sees that in the standard ordering on sequents in every rule every premiss is of lower complexity than its conclusion, except in the left implication rule, where the principle implication occurs in the left premiss of the rule. This explains why the only difference between G4ip and G3ip is the left implication rule(s): the one left implication rule of G3ip is in G4ip replaced by four rules, each corresponding to the outermost connective of the antecedent of the principal implication.

We consider the following modal and additional left implication rules, where $\Pi$ ranges over multisets that do not contain boxed formulas:

$$
\frac{\Gamma \Rightarrow \varphi}{\Pi, \square \Gamma \Rightarrow \square \varphi} \mathcal{R}_{K} \quad \frac{\Gamma, \varphi \Rightarrow}{\Pi, \square \Gamma, \square \varphi \Rightarrow \Delta} \mathcal{R}_{D} \quad \frac{\Gamma \Rightarrow \varphi \quad \Pi, \square \Gamma, \psi \Rightarrow \Delta}{\Pi, \square \Gamma, \square \varphi \rightarrow \psi \Rightarrow \Delta} L \square \rightarrow
$$




$$
\begin{array}{ll}
\Gamma, p \Rightarrow p \quad \text { At } \quad(p \text { an atom }) & \Gamma, \perp \Rightarrow \Delta \quad L \perp \\
\frac{\Gamma \Rightarrow \varphi \quad \Gamma \Rightarrow \psi}{\Gamma \Rightarrow \varphi \wedge \psi} R \wedge & \frac{\Gamma, \varphi, \psi \Rightarrow \Delta}{\Gamma, \varphi \wedge \psi \Rightarrow \Delta} L \wedge \\
\frac{\Gamma \Rightarrow \varphi_{i}}{\Gamma \Rightarrow \varphi_{0} \vee \varphi_{1}} R \vee(i=0,1) & \frac{\Gamma, \varphi \Rightarrow \Delta \quad \Gamma, \psi \Rightarrow \Delta}{\Gamma, \varphi \vee \psi \Rightarrow \Delta} L \vee \\
\frac{\Gamma, \varphi \Rightarrow \psi}{\Gamma \Rightarrow \varphi \rightarrow \psi} R \rightarrow & \frac{\Gamma, p, \varphi \Rightarrow \Delta}{\Gamma, p, p \rightarrow \varphi \Rightarrow \Delta} L p \rightarrow(p \text { an atom }) \\
\frac{\Gamma, \varphi \rightarrow(\psi \rightarrow \gamma) \Rightarrow \Delta}{\Gamma, \varphi \wedge \psi \rightarrow \gamma \Rightarrow \Delta} L \wedge \rightarrow & \frac{\Gamma, \varphi \rightarrow \gamma, \psi \rightarrow \gamma \Rightarrow \Delta}{\Gamma, \varphi \vee \psi \rightarrow \gamma \Rightarrow \Delta} L \vee \rightarrow \\
\frac{\Gamma, \psi \rightarrow \gamma \Rightarrow \varphi \rightarrow \psi}{\Gamma,(\varphi \rightarrow \psi) \rightarrow \gamma \Rightarrow \Delta} \quad &
\end{array}
$$

Figure 2.1: The Gentzen calculus G4ip

$$
\begin{array}{ll}
\Gamma, p \Rightarrow p \quad \text { At } \quad(p \text { an atom }) & \Gamma, \perp \Rightarrow \Delta \quad L \perp \\
\frac{\Gamma \Rightarrow \varphi \quad \Gamma \Rightarrow \psi}{\Gamma \Rightarrow \varphi \wedge \psi} R \wedge & \frac{\Gamma, \varphi, \psi \Rightarrow \Delta}{\Gamma, \varphi \wedge \psi \Rightarrow \Delta} L \wedge \\
\frac{\Gamma \Rightarrow \varphi_{i}}{\Gamma \Rightarrow \varphi_{0} \vee \varphi_{1}} R \vee(i=0,1) & \frac{\Gamma, \varphi \Rightarrow \Delta \quad \Gamma, \psi \Rightarrow \Delta}{\Gamma, \varphi \vee \psi \Rightarrow \Delta} L \vee \\
\frac{\Gamma, \varphi \Rightarrow \psi}{\Gamma \Rightarrow \varphi \rightarrow \psi} R \rightarrow & \frac{\Gamma, \varphi \rightarrow \psi \Rightarrow \varphi \quad \Gamma, \psi \Rightarrow \Delta}{\Gamma, \varphi \rightarrow \psi \Rightarrow \Delta} L \rightarrow
\end{array}
$$

Figure 2.2: The Gentzen calculus G3ip 


\begin{tabular}{l|l|l|l|l|l} 
& G3ip & G4ip & $L \square \rightarrow$ & $\mathcal{R}_{K}$ & $\mathcal{R}_{D}$ \\
\hline G3iK $_{\square}$ & + & & & + & \\
\hline G4iK $_{\square}$ & & + & + & + & \\
\hline G3iKD $_{\square}$ & + & & & + & + \\
\hline G4iKD $_{\square}$ & & + & + & + & +
\end{tabular}

Figure 2.3: The calculi of the form $\mathrm{G}_{3} \mathrm{X}_{\square}$ and $\mathrm{G} 4 \mathrm{i} \mathrm{X}_{\square}$ for $\mathrm{X} \in\{\mathrm{K}, \mathrm{KD}\}$.

The calculus $\mathrm{G}_{\mathrm{iK}} \square$ consists of the calculus G3ip, but then for the modal language, plus the rule $\mathcal{R}_{K}$. G4iK $\square$ consists of the calculus G4ip, but then for the modal language, plus the rules $L \square \rightarrow$ and $\mathcal{R}_{K}$. G4iKD $\square$ consists of $\mathrm{G} \mathrm{iK}_{\square}$ plus the rule $\mathcal{R}_{D}$. See Figure 2.3 for a summary of the above in a table. Note that in the name of the calculi we have suppressed the $p$ that indicates that we are dealing with a propositional logic, in order to have names of decent length.

In the rules of $\mathrm{G}_{3} \mathrm{X}_{\square}$, the principal formula of an inference is defined as usual for the connectives, in the modal rules $\mathcal{R}_{K}$ and $\mathcal{R}_{D}$ all formulas in $\square \Gamma$ as well as $\square \varphi$ are principal, and in the rule $L \square \rightarrow$ all formulas in $\square \Gamma$ and $\square \varphi \rightarrow \psi$ are principal.

The depth of a proof is the length of its longest branch. If $\vdash$ stands for derivability in a given calculus, the we write $\vdash_{d} S$ if $S$ has a proof of depth at most $d$ in that calculus.

\section{Structural rules in $\mathrm{G}_{3} \mathrm{X}_{\square}$}

Lemma 1 (Weakening, Contraction, Inversion Lemma)

For $\mathrm{X} \in\{\mathrm{K}, \mathrm{KD}, \mathrm{K} 4\}$ and $\vdash$ denoting $\vdash_{\mathrm{G}_{3} \mathrm{X}_{\square}}$, the following statements hold.

Falsum rule $\quad \vdash_{d} \Gamma \Rightarrow \perp$ implies $\vdash_{d} \Gamma \Rightarrow \Delta$.

Weakening $\quad \vdash_{d} \Gamma \Rightarrow \Delta$ implies $\vdash_{d} \Gamma, \varphi \Rightarrow \Delta$.

Inversion $\wedge \quad \vdash_{d} \Gamma, \varphi \wedge \psi \Rightarrow \Delta$ implies $\vdash_{d} \Gamma, \varphi, \psi \Rightarrow \Delta$.

Inversion $\vee \quad \vdash_{d} \Gamma, \varphi_{1} \vee \varphi_{2} \Rightarrow \Delta$ implies $\vdash_{d} \Gamma, \varphi_{i} \Rightarrow \Delta$ for $i=1,2$.

Inversion $R \rightarrow \vdash_{d} \Gamma \Rightarrow \varphi \rightarrow \psi$ implies $\vdash_{d} \Gamma, \varphi \Rightarrow \psi$.

Inversion $L \rightarrow \quad \vdash_{d} \Gamma, \varphi \rightarrow \psi \Rightarrow \Delta$ implies $\vdash_{d} \Gamma, \psi \Rightarrow \Delta$.

Contraction $\quad \vdash_{d} \Gamma, \varphi, \varphi \Rightarrow \Delta$ implies $\vdash_{d} \Gamma, \varphi \Rightarrow \Delta$.

Weakening also holds when $\vdash$ denotes $\vdash_{\mathrm{G} 4 \mathrm{i}} \mathrm{X}_{\square}$.

Proof The proofs of all properties except Weakening are standard and therefore omitted. For details, see page 66-67 in (Troelstra and Schwichtenberg, 1996). 
Weakening is different because of the extra requirement on contexts in the modal rules, and it can be proved as follows with induction to $d$. We treat the case that $\vdash$ denotes $\vdash_{\mathrm{G}_{3 \mathrm{i}} \mathrm{X}_{\square}}$, the case that it denotes $\vdash_{\mathrm{G}_{4} \mathrm{X} \mathrm{X}_{\square}}$ is similar. Thus suppose $\vdash_{d} S$. If $S$ is an instance of an axiom, the statement clearly holds. If the last inference in the proof is not a modal rule, the proof that $\vdash_{d} \Gamma, \varphi \Rightarrow \Delta$ is as usual. Therefore suppose the last inference is $\mathcal{R}_{K}$, for $\mathcal{R}_{D}$ the reasoning is similar. Thus $S=(\Pi, \square \Gamma \Rightarrow \square \psi)$ and the premiss is $\Gamma \Rightarrow \psi$. We have to show that $\vdash_{d} \Pi, \square \Gamma, \varphi \Rightarrow \square \psi$. If $\varphi$ is not a boxed formula, this follows immediately from an application of $\mathcal{R}_{K}$ to the premiss. In case $\varphi=\square \chi$, then we use that by the induction hypothesis $\vdash_{d-1} \Gamma, \chi \Rightarrow \psi$. Thus $\vdash_{d} \Pi, \square \Gamma, \square \chi \Rightarrow \square \psi$ follows. $\quad \dashv$

\subsection{Admissibility of Cut}

We consider the multiplicative version of Cut,

$$
\frac{\Gamma_{l} \Rightarrow \varphi \quad \Gamma_{r}, \varphi \Rightarrow \psi}{\Gamma_{l}, \Gamma_{r} \Rightarrow \psi} \text { Cut }
$$

and show that it is admissible in $\mathrm{GBiK}_{\square}$ and $\mathrm{G} 3 \mathrm{iK} \mathrm{D}_{\square}$. This fact is used to prove their equivalence to $\mathrm{G}_{4} \mathrm{iK}_{\square}$ and $\mathrm{G} 4 \mathrm{iK} \mathrm{D}_{\square}$, respectively.

Given a proof in $\mathrm{G}_{3 \mathrm{i}} \mathrm{X}_{\square}$, the level of a cut is the sum of the depths of the deductions of the premisses, and the rank of a cut with cutformula $\varphi$ is $|\varphi|$, where $|\varphi|$ denotes the maximum length of a branch in its construction tree. The cutrank of a proof is the maximum of the ranks of the cuts occurring in the proof.

Theorem 1 (Cut Admissibility Lemma)

The Cut Rule is admissible in $\mathrm{G} \mathrm{iK}_{\square}$ and $\mathrm{G}_{3} \mathrm{iKD} \mathrm{D}_{\square}$.

Proof Let $\vdash$ denote $\vdash_{\mathrm{G}_{3 i X}}$. Following the corrected version (Troelstra, 1998) of the cut elimination proof for G3ip in (Troelstra and Schwichtenberg, 1996), we successively eliminate cuts from the proof, always considering those cuts that have no cuts above them, the topmost cuts. For this it suffices to show that for cutfree proofs $\mathcal{D}_{l}$ and $\mathcal{D}_{r}$ the following proof $\mathcal{D}$ can be transformed into a cutfree proof of the same endsequent.

$$
\frac{\begin{array}{c}
\mathcal{D}_{l} \\
\Gamma_{l} \Rightarrow \varphi
\end{array} \quad \begin{array}{c}
\mathcal{D}_{r} \\
\Gamma_{r}, \varphi \Rightarrow \Delta
\end{array}}{\Gamma_{l}, \Gamma_{r} \Rightarrow \Delta}
$$

This is proved by induction on the cutrank with a subinduction on the level of the cut. We use the fact that $\mathrm{G}_{3} \mathrm{iX}_{\square}$ is closed under weakening and contraction implicitly at various places.

There are three possibilities:

1. at least one of the premisses is an axiom; 
2. both premisses are not axioms and the cutformula is not principal in at least one of the premisses;

3. the cutformula is principal in both premisses, which are not axioms.

1. As in (Troelstra and Schwichtenberg, 1996), straightforward, by checking all possible cases.

2. First, the case that $\varphi$ is not principal in $\mathcal{D}_{l}$. Thus the last inference in $\mathcal{D}_{l}$ is $\mathcal{R}_{D}$, if present, or one of the nonmodal rules of $\mathrm{G} 3 \mathrm{X}_{\square}$. In the first case, $\Gamma_{l}=\Pi, \square \Gamma^{\prime}, \square \psi$ and the last inference is of the form

$$
\begin{aligned}
& \mathcal{D}^{\prime} \\
& \frac{\Gamma^{\prime}, \psi \Rightarrow}{\Pi, \square \Gamma^{\prime}, \square \psi \Rightarrow \varphi} \quad \Gamma_{r}, \varphi \Rightarrow \Delta \\
& \Gamma_{l}, \Gamma_{r} \Rightarrow \Delta
\end{aligned}
$$

Clearly, the following is a cutfree derivation of the sequent $\Pi, \square \Gamma^{\prime}, \square \psi \Rightarrow \Delta$ :

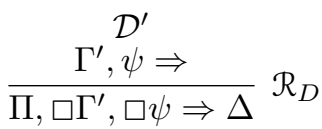

By applications of Weakening (Lemma 1) this derivation can be made into a cutfree derivation of $\Gamma_{r}, \Pi, \square \Gamma^{\prime}, \square \psi \Rightarrow \Delta$.

In case the last inference in $\mathcal{D}_{l}$ is not a modal rule, then if $\mathcal{R}$ is a two premiss rule it can only be $\mathrm{L} \vee$ or $\mathrm{L} \rightarrow$. In the first case the last part of the proof looks like

$$
\frac{\Gamma, \psi \Rightarrow \varphi \quad \Gamma, \psi^{\prime} \Rightarrow \varphi}{\Gamma, \psi \vee \psi^{\prime} \Rightarrow \varphi} \mathcal{R} \begin{gathered}
\mathcal{D}_{r} \\
\Gamma, \varphi \Rightarrow \Delta
\end{gathered}
$$

where $\Gamma_{l}, \Gamma_{r}=\Gamma, \Gamma^{\prime}, \psi \vee \psi^{\prime}$. The cut can be pushed upwards in the following way.

$$
\frac{\Gamma, \psi \Rightarrow \varphi \quad \Gamma^{\prime}, \varphi \Rightarrow \Delta}{\frac{\Gamma, \Gamma^{\prime}, \psi \Rightarrow \Delta}{\Gamma, \Gamma^{\prime}, \psi \vee \psi^{\prime} \Rightarrow \Delta}} \frac{\Gamma, \psi^{\prime} \Rightarrow \varphi \quad \Gamma^{\prime}, \varphi \Rightarrow \Delta}{\Gamma, \Gamma^{\prime}, \psi^{\prime} \Rightarrow \Delta} \mathcal{R}
$$

Thus we obtain a proof of $\Gamma_{l}, \Gamma_{r} \Rightarrow \Delta$ with cuts of the same rank as the cut in $\mathcal{D}$ but of lower level, and the induction hypothesis can be applied. In case $\mathcal{R}$ is $\mathrm{L} \rightarrow$, the last part of the proof looks like

$$
\begin{gathered}
\frac{\Gamma, \psi \rightarrow \psi^{\prime} \Rightarrow \psi \quad \Gamma, \psi^{\prime} \Rightarrow \varphi}{\Gamma, \psi \rightarrow \psi^{\prime} \Rightarrow \varphi} \mathcal{R} \begin{array}{c}
\mathcal{D}_{r} \\
\Gamma^{\prime}, \varphi \Rightarrow \Delta
\end{array} \\
\hline, \Gamma^{\prime}, \psi \rightarrow \psi^{\prime} \Rightarrow \Delta
\end{gathered}
$$

where $\Gamma_{l}, \Gamma_{r}=\Gamma, \Gamma^{\prime}, \psi \rightarrow \psi^{\prime}$. Also in this case the cut can be pushed upwards (double lines suppress applications of weakening):

$$
\frac{\frac{\Gamma, \psi \rightarrow \psi^{\prime} \Rightarrow \psi}{\overline{\Gamma, \Gamma^{\prime}, \psi \rightarrow \psi^{\prime} \Rightarrow \psi}} \frac{\Gamma, \psi^{\prime} \Rightarrow \varphi \quad \Gamma^{\prime}, \varphi \Rightarrow \Delta}{\Gamma, \Gamma^{\prime}, \psi^{\prime} \Rightarrow \Delta}}{\Gamma, \Gamma^{\prime}, \psi \rightarrow \psi^{\prime} \Rightarrow \Delta} \mathcal{R}
$$


Thus we obtain a proof of $\Gamma_{l}, \Gamma_{r} \Rightarrow \Delta$ with a cut of the same rank as the cut in $\mathcal{D}$ but of lower level, and the induction hypothesis can be applied. One premiss rules can be treated in a similar way.

Second, the case that $\varphi$ is not principal in $\mathcal{D}_{r}$. The nonmodal rules are handled as in the previous case. We treat the case that it is an application of $\mathcal{R}_{K}, \mathcal{R}_{D}$ can be treated in the same way. Thus the lower part of $\mathcal{D}$ looks as follows.

$$
\frac{\mathcal{D}^{\prime}}{\frac{\Gamma \stackrel{\Rightarrow}{\Rightarrow} \Rightarrow}{\Gamma_{l} \Rightarrow \varphi(\square \Gamma, \varphi \Rightarrow \square \psi}} \mathcal{R}_{K}
$$

Suppose $\Gamma_{l}=\Pi^{\prime}, \square \Gamma^{\prime}$, where $\Pi^{\prime}$ does not contain boxed formulas. Clearly, the following is a cutfree proof of the same endsequent.

$$
\frac{\mathcal{D}^{\prime}}{\Gamma^{\prime}, \Gamma \Rightarrow \psi} \mathcal{R}_{K}
$$

3. The cutformula is principal in both premisses, which are not axioms. We distinguish by cases according to the form of the cutformula, and treat implications and boxed formulas.

If the cutformula is an implication, the last inference looks as follows.

$$
\frac{\begin{array}{c}
\mathcal{D}_{1} \\
\frac{\Gamma_{l}, \varphi \Rightarrow \psi}{\Gamma_{l} \Rightarrow \varphi \rightarrow \psi} R \rightarrow \quad \frac{\mathcal{D}_{r}, \varphi \rightarrow \psi \Rightarrow \varphi}{\Gamma_{r}, \psi}
\end{array} \quad \begin{array}{c}
\mathcal{D}_{3} \\
\Gamma_{r}, \varphi \rightarrow \psi
\end{array}}{\Gamma_{l}, \Gamma_{r} \Rightarrow \Delta} L \rightarrow
$$

This is replaced by the proof

$$
\begin{aligned}
& \mathcal{D}_{1}
\end{aligned}
$$

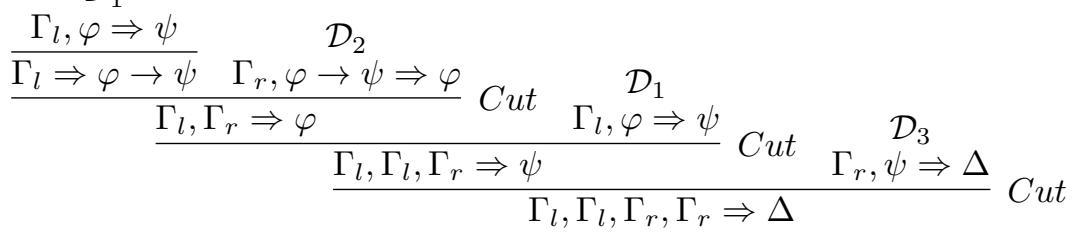

in which each cut either is of lower rank or of the same rank but of lower level as the cut in $\mathcal{D}$. The other connectives can be treated in the same way.

If the cutformula is a boxed formula, then the form of the last inference depends on which modal rules are present in the calculus. The fact that the cutformula is boxed and principal in the last inference of both $\mathcal{D}_{l}$ and $\mathcal{D}_{r}$ implies that there are only two possible combinations for these last inferences: both are (instances of) $\mathcal{R}_{K}$ or the last inference of $\mathcal{D}_{l}$ is $\mathcal{R}_{K}$ and that of $\mathcal{D}_{r}$ is $\mathcal{R}_{D}$. 
First, suppose the last inference has the following form.

$$
\begin{aligned}
& \mathcal{D}_{1} \quad \mathcal{D}_{2} \\
& \frac{\frac{\Gamma_{1} \Rightarrow \varphi}{\Pi_{1}, \square \Gamma_{1} \Rightarrow \square \varphi} \mathcal{R}_{K} \quad \frac{\Gamma_{2}, \varphi \Rightarrow \psi}{\Pi_{2}, \square \Gamma_{2}, \square \varphi \Rightarrow \square \psi}}{\Pi_{1}, \Pi_{2}, \square \Gamma_{1}, \square \Gamma_{2} \Rightarrow \square \psi} \mathcal{R}_{K}
\end{aligned}
$$

This can be replaced by a proof of lower cutrank, namely

$$
\begin{gathered}
\mathcal{D}_{1} \quad \begin{array}{c}
\mathcal{D}_{2} \\
\Gamma_{1} \Rightarrow \varphi \quad \Gamma_{2}, \varphi \Rightarrow \psi \\
\Gamma_{1}, \Gamma_{2} \Rightarrow \psi
\end{array} \\
\frac{\Pi_{1}, \Pi_{2}, \square \Gamma_{1}, \square \Gamma_{2} \Rightarrow \square \psi}{}
\end{gathered}
$$

The same argument applies when the last inference of $\mathcal{D}_{r}$ is $\mathcal{R}_{D}$.

\section{Strict proofs in $\mathrm{G}_{4 \mathrm{i}}$}

In this section we prove a normal form theorem for proofs in the calculi G3iX which enables us to establish, in the next section, that $\mathrm{G}_{3} \mathrm{iX}_{\square}$ and $\mathrm{G} 4 \mathrm{i} \mathrm{X}_{\square}$ are equivalent, meaning that they derive the same sequents. This in turn implies that the structural rules are admissible in the latter, a fact that is used in the application of these calculi in the context of uniform interpolation in (Iemhoff, 2018b).

A multiset is irreducible if it has no element that is a disjunction or a conjunction or falsum and for no atom $p$ does it contain both $p \rightarrow \psi$ and $p$. A sequent $S$ is irreducible if $S^{a}$ is. A proof is sensible if the principal formula of the last inference is not of the form $p \rightarrow \psi$ for some atom $p$. A proof in $\mathrm{G}_{3} \mathrm{iX}$ is strict if in the last inference, in case it is an instance of $\mathrm{L} \rightarrow$ with principal formula $\square \varphi \rightarrow \psi$, the left premiss is an axiom or the conclusion of an application of the modal rule $\mathcal{R}_{K}$. Note that because the formula in the succedent of the left premiss is $\square \varphi$, in case the left premiss is an instance of an axiom, it can only be an instance of $L \perp$. This implies that if $S$ is irreducible, the left premiss cannot be an instance of an axiom and thus is required to be the conclusion of an application of $\mathcal{R}_{K}$.

Lemma 2 For $\mathrm{X} \in\{\mathrm{K}, \mathrm{KD}\}$ : Every irreducible sequent that is provable in $\mathrm{G} \mathrm{iX}_{\square}$ has a sensible strict proof.

Proof This is proved in the same way as the corresponding lemma (Lemma 1) in (Dyckhoff, 1992). Arguing by contradiction, assume that among all provable irreducible sequents that have no sensible strict proofs, $S$ is such a sequent with the shortest proof, $\mathcal{D}$, where the length of a proof is the length of its leftmost branch. Thus the last inference in the proof is an application

$$
\begin{array}{cc}
\mathcal{D}_{l} & \mathcal{D}_{r} \\
\Gamma, \varphi \rightarrow \psi \Rightarrow \varphi & \Gamma, \psi \Rightarrow \Delta
\end{array}
$$


of $\mathrm{L} \rightarrow$, where $\varphi$ is an atom or a boxed formula. Since $S^{a}$ is irreducible, $\perp \notin S^{a}$ and if $\varphi$ is an atom, $\varphi \notin S^{a}$. Therefore the left premiss cannot be an axiom and hence is the conclusion of a rule, say $\mathcal{R}$. Since the succedent of the conclusion of $\mathcal{R}$ consists of an atom or a boxed formula, $\mathcal{R}$ is a left rule or a modal rule. $\mathcal{R}$ cannot be $\mathcal{R}_{K}$ because then the proof would be strict and sensible. Thus it is a left rule or $\mathcal{R}_{D}$.

In the last case, for some $\Pi, \Gamma^{\prime}$ such that $\Gamma=\Pi, \square \Gamma^{\prime}$ the last part of the proof looks as follows.

$$
\begin{array}{cc}
\begin{array}{c}
\mathcal{D}^{\prime} \\
\Gamma^{\prime} \Rightarrow
\end{array} & \mathcal{D}_{r} \\
\frac{\prod, \square \Gamma^{\prime}, \varphi \rightarrow \psi \Rightarrow \varphi}{\Pi, \square \Gamma^{\prime}, \varphi \rightarrow \psi \Rightarrow \Delta} & \mathcal{R}_{D} \quad \Pi, \square \Gamma^{\prime}, \psi \Rightarrow \Delta
\end{array}
$$

Hence the following is a sensible strict proof of the same endsequent.

$$
\frac{\mathcal{D}^{\prime}}{\Pi, \square \Gamma^{\prime}, \varphi \rightarrow \psi \Rightarrow \Delta} \mathcal{R}_{D}
$$

We turn to the case that $\mathcal{R}$ is not a modal rule and proceed as in (Dyckhoff, 1992). Sequent $(\Gamma, \varphi \rightarrow \psi \Rightarrow \varphi)$ is irreducible and has a shorter proof than $S$. Thus its subproof $\mathcal{D}_{l}$ is strict and sensible. Since the sequent is irreducible and $\varphi$ is an atom or a boxed formula, the last inference of $\mathcal{D}_{l}$ is $\mathrm{L} \rightarrow$ with a principal formula $\varphi^{\prime} \rightarrow \psi^{\prime}$ such that $\varphi^{\prime}$ is not an atom. Let $\mathcal{D}^{\prime}$ be the proof of the left premiss $\left(\Gamma, \varphi \rightarrow \psi \Rightarrow \varphi^{\prime}\right)$. Thus the last part of $\mathcal{D}$ looks as follows, where $\Pi, \varphi^{\prime} \rightarrow \psi^{\prime}=\Gamma$.

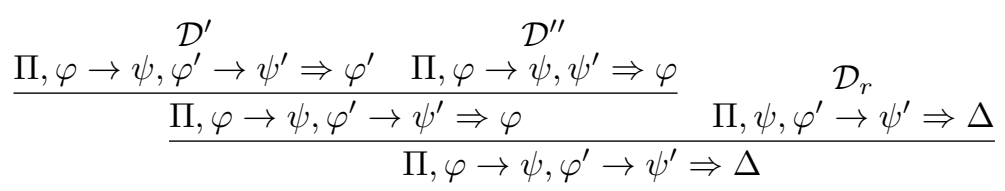

Consider the following proof of $S$.

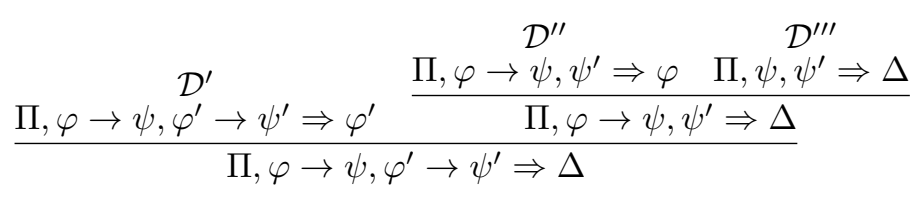

The existence of $\mathcal{D}^{\prime \prime \prime}$ follows from the Inversion Lemma and the existence of $\mathcal{D}_{r}$. The obtained proof is strict and sensible: In case $\varphi^{\prime}$ is not a boxed formula, this is straightforward. In case $\varphi^{\prime}$ is a boxed formula, it follows from the fact, observed above, that $\mathcal{D}_{l}$ is strict and sensible.

\section{Equivalence of $\mathrm{G}_{3 \mathrm{i}} \mathrm{X}_{\square}$ and $\mathrm{G}_{4 \mathrm{i}} \mathrm{X}_{\square}$}

Theorem 2 For $\mathrm{X} \in\{\mathrm{K}, \mathrm{KD}\}: \vdash_{\mathrm{G}_{3} \mathrm{X} \mathrm{X}_{\square}} S$ if and only if $\vdash_{\mathrm{G} 4 \mathrm{i} \mathrm{X}_{\square}} S$. 
Proof The proof is an adaptation of the proof of Theorem 1 in (Dyckhoff, 1992). Let $\mathrm{X} \in\{\mathrm{K}, \mathrm{KD}\}$.

First, the proof of the direction from right to left. This is straightforward because $\mathrm{G}_{3 \mathrm{iX}}$ is closed under the structural rules and $C u t$, but let's fill in some of the details. We use induction to the depth of the proof of a sequent in $\mathrm{G}_{4} \mathrm{XX}_{\square}$. Suppose $\vdash_{\mathrm{G}_{4} \mathrm{X}}{ }_{\square} S$. If $S$ is an instance of an axiom, then clearly $\vdash_{\mathrm{G} 3 \mathrm{i}} \square S$ as well. Suppose $S$ is not an instance of an axiom and consider the last inference of the proof of $S$. We distinguish according to the rule $\mathcal{R}$ of which the last inference is an instance.

If the rule is $L p \rightarrow$, then $S$ is of the form $\Gamma, p, p \rightarrow \varphi \Rightarrow \Delta$. The premiss is $\Gamma, p, \varphi \Rightarrow \Delta$, which, by the induction hypothesis, is derivable in $\mathrm{G} 3 \mathrm{X}_{\square}$. It is not hard to show that $\Gamma, p, p \rightarrow \varphi \Rightarrow \varphi$ is also derivable in $\mathrm{G} \mathrm{X}_{\square}$. Applications of Cut and Contraction show that so is $S$.

If the rule is $L \rightarrow$, then $S$ is of the form $\Gamma,(\varphi \rightarrow \psi) \rightarrow \gamma \Rightarrow \Delta$ and the premisses are $\Gamma, \gamma \Rightarrow \Delta$ and $\Gamma, \psi \rightarrow \gamma \Rightarrow \varphi \rightarrow \psi$. The premisses are derivable in $\mathrm{G}_{3} \mathrm{X}_{\square}$ by the induction hypothesis. It is not difficult to show that then $\Gamma,(\varphi \rightarrow \psi) \rightarrow \gamma, \varphi \Rightarrow \psi$ is derivable in $\mathrm{G}_{3} \mathrm{IX}_{\square}$ as well. Hence so is $\Gamma,(\varphi \rightarrow \psi) \rightarrow \gamma \Rightarrow \varphi \rightarrow \psi$. An application of $L \rightarrow$ proves that $S$ is derivable in $\mathrm{G}_{\mathrm{i}} \mathrm{X}_{\square}$.

The remaining cases are left to the reader.

The other direction is proved by induction on the well-ordering $\ll$ defined in Section 2. Consider a sequent $S$ provable in $\mathrm{G} \mathrm{iX} \mathrm{X}_{\square}$. Since $\mathrm{G} \mathrm{i} \mathrm{X}_{\square}$ and $\mathrm{G} 4 \mathrm{i}_{\square}$ have the same axioms, the case that $S$ is an instance of an axiom is trivial. Therefore assume this is not the case. If $S^{a}$ contains a conjunction, $S=\left(\Gamma, \varphi_{1} \wedge \varphi_{2} \Rightarrow \Delta\right)$, then $\Gamma, \varphi_{1}, \varphi_{2} \Rightarrow \Delta$ is provable in $\mathrm{G} \mathrm{iX} \mathrm{X}_{\square}$ too by the Inversion Lemma. Hence it is provable in $\mathrm{G}_{4} \mathrm{IX}_{\square}$ by the induction hypothesis. Thus so is $\Gamma, \varphi_{1} \wedge \varphi_{2} \Rightarrow \Delta$. A disjunction in $S^{a}$ as well as the case that both $p$ and $p \rightarrow \varphi$ belong to $S^{a}$, can be treated in the same way.

Thus only the case that $S$ is irreducible remains, and by Lemma 2 we may assume its proof to be sensible and strict. Thus the last inference is an application of a rule, $\mathcal{R}$, that is or is not equal to $L \rightarrow$. In the last case, $\mathcal{R}$ belongs to both calculi and the premiss of $\mathcal{R}$ is lower in the well-ordering $\ll$ than $S$, and thus the induction hypothesis applies. In the first case, suppose that the principal formula of the last inference is $\gamma \rightarrow \psi$ and $S=(\Gamma, \gamma \rightarrow \psi \Rightarrow \Delta)$. Since the proof is sensible, $\gamma$ is not atomic. We distinguish according to the form of $\gamma$.

If $\gamma=\perp$, then $\Gamma \Rightarrow \Delta$ is derivable in $\mathrm{G} 3 \mathrm{X}_{\square}$, and therefore in $\mathrm{G}_{4} \mathrm{iX} \mathrm{X}_{\square}$. As $\mathrm{G}_{\mathrm{i}} \mathrm{X}_{\square}$ is closed under weakening, Lemma $1, S$ is derivable in $\mathrm{G}_{4 \mathrm{iX}} \mathrm{X}_{\square}$ too.

If $\gamma=\varphi_{1} \wedge \varphi_{2}$, then $\Gamma, \varphi_{1} \rightarrow\left(\varphi_{2} \rightarrow \psi\right) \Rightarrow \Delta$ is derivable in $\mathrm{GBX}_{\square}$. Thus the sequent is derivable in $\mathrm{G}_{4} \mathrm{iX} \square$ by the induction hypothesis. Hence so is $\Gamma, \varphi_{1} \wedge \varphi_{2} \rightarrow \psi \Rightarrow \Delta$.

If $\gamma=\varphi_{1} \vee \varphi_{2}$, then $\Gamma, \varphi_{1} \rightarrow \psi, \varphi_{2} \rightarrow \psi \Rightarrow \Delta$ is derivable in $\mathrm{G} \mathrm{iX} \mathrm{X}_{\square}$. Thus the sequent is derivable in $\mathrm{G}_{4} \mathrm{iX} \mathrm{X}_{\square}$ by the induction hypothesis. Hence so is $\Gamma, \varphi_{1} \vee \varphi_{2} \rightarrow \psi \Rightarrow \Delta$. 
If $\gamma=\varphi_{1} \rightarrow \varphi_{2}$, then because $\gamma \rightarrow \psi$ is the principal formula, both sequents $\Gamma, \gamma \rightarrow \psi \Rightarrow \gamma$ and $\Gamma, \psi \Rightarrow \Delta$ are derivable in $\mathrm{G} \mathrm{IX}_{\square}$. Thus so is sequent $\Gamma, \varphi_{2} \rightarrow \psi \Rightarrow \varphi_{1} \rightarrow \varphi_{2}$. Since this sequent and $\Gamma, \psi \Rightarrow \Delta$ are lower in complexity than $S$, they are derivable in $\mathrm{G} \mathrm{iX}_{\square}$ by the induction hypothesis. Hence so is $S$.

If $\gamma=\square \varphi$, the fact that the proof is strict implies that the left premiss, $(\Gamma, \square \varphi \rightarrow \psi \Rightarrow \square \varphi)$, is the conclusion of an application of $\mathcal{R}_{K}$ to some sequent $\left(\Gamma^{\prime} \Rightarrow \varphi\right)$. Thus $\square \Gamma^{\prime} \subseteq \Gamma$. By the induction hypothesis, $\Gamma^{\prime} \Rightarrow \varphi$ and the right premiss $(\Gamma, \psi \Rightarrow \Delta)$ are derivable in $\mathrm{G}_{4} \mathrm{X}_{\square}$, say with proofs $\mathcal{D}$ and $\mathcal{D}^{\prime}$, respectively. And since $L \square \rightarrow$ belongs to the calculus, the following is a proof of $(\Gamma, \square \varphi \rightarrow \psi \Rightarrow \Delta)$ in $\mathrm{G}_{4} \mathrm{iX}_{\square}$,

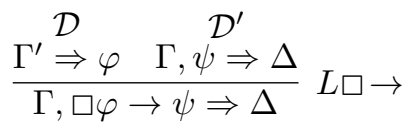

From the previous theorem and Lemma 1 the following can be obtained.

Corollary 1 The rules Cut, Weakening, and Contraction are admissible in $\mathrm{G}_{4} \mathrm{KK}_{\square}$ and $\mathrm{G} 4 \mathrm{iKD}_{\square}$.

\section{Termination}

A sequent calculus is terminating if backwards proof search always terminates, that is, if the process that starts with a given sequent and applies the rules of the calculus backwards in whatever order as long as possible, always in a finite number of steps reaches the point where no new sequents can be obtained anymore.

Clearly, the calculi $\mathrm{G}_{4} \mathrm{iK}_{\square}$ and $\mathrm{G} \mathrm{iK \textrm {KD } _ { \square }}$ are terminating, since in every rule the conclusion is higher in the order $\ll$ on sequents than the premisses.

Theorem 3 The calculi $\mathrm{G} 4 \mathrm{iK}_{\square}$ and $\mathrm{G} 4 \mathrm{iK}_{\square}$ are terminating sequent calculi for the intuitionistic modal logics $\mathrm{L}_{\mathrm{G} 4 \mathrm{~K}_{\square}}$ and $\mathrm{L}_{\mathrm{G}_{4} \mathrm{~K}_{\square}}$, respectively, and the cut rule and the structural rules are admissible in them.

\section{Conclusion}

It has been shown that for $\mathrm{X} \in\{\mathrm{K}, \mathrm{KD}\}$ the calculus $\mathrm{G} 4 \mathrm{i} \mathrm{X}_{\square}$ introduced in this paper is a terminating cutfree sequent calculus for the intuitionistic modal logic $\mathrm{L}_{G 3 i X_{\square}}$ in which the structural rules are admissible. The result is obtained via a syntactic proof of the equivalence between $\mathrm{G}_{4} \mathrm{XX}_{\square}$ and the calculus $\mathrm{G}_{\mathrm{i}} \mathrm{X}_{\square}$. One wonders whether this equivalence can be proved in another way. One could, for example, try to establish in a semantic way that $\mathrm{G}_{4} \mathrm{IX}_{\square}$ is sound and complete with respect to a semantics to which $\mathrm{G}_{3} \mathrm{iX}_{\square}$ is sound and complete as well. Since the proofs in the paper are all more or less straightforward, it is not 
very likely that in this particular case such a semantic approach would be more straightforward, but it may, of course, have other benefits.

\subsection{Other intuitionistic modal logics}

In this section we compare the logics in this paper to related logics in the literature.

In his thesis Simpson (1994) formulates six requirements that an intuitionistic modal logic should obey. Both logics $\mathrm{L}_{G}{ }_{\mathrm{Gi}} \mathrm{K}_{\square}$ and $\mathrm{L}_{\mathrm{G}_{3 i} \mathrm{~K}_{\square}}$ in this paper satisfy the first four requirements:

$\circ \mathrm{L}_{\mathrm{G} 3 \mathrm{i}} \mathrm{X}_{\square}$ is conservative over IPC;

- $\mathrm{L}_{\mathrm{G} 3 \mathrm{i}} \mathrm{X}_{\square}$ contains all substitution instances of theorems of IPC and is closed under modus ponens;

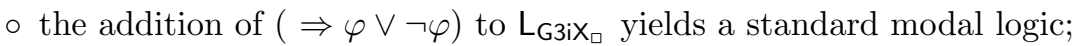

○ if $\varphi \vee \psi$ holds in $\mathrm{L}_{\mathrm{G} 3 i X_{\square}}$, then either $\varphi$ or $\psi$ holds in $\mathrm{L}_{\mathrm{G} 3 i \mathrm{X}_{\square}}$.

The last two requirements from (Simpson, 1994) do not apply as they are about the diamond operator and the semantics.

In (Simpson, 1994), Figure 3-3 on page 46, the intuitionistic modal logic Intuitionistic $K$ without $\diamond$ is defined by the Hilbert system given by the rules Modus Ponens and Necessitation, the $K$-axiom $\square(\varphi \rightarrow \psi) \rightarrow(\square \varphi \rightarrow \square \psi)$ and all theorems of IPC. From this axiomatization on the one hand and the admissibility of cut and Lemma 1 for $\mathrm{L}_{\mathrm{G}_{3} \mathrm{~K}_{\square}}$ on the other hand, plus the fact that all theorems of IPC hold in both logics, it follows that the two logics are the same. In a similar way it can be established that the logics with diamond $I K$ and $I K D$ from (Simpson, 1994), Figures 3-6 and 3-7, contain $\mathrm{L}_{\mathrm{G}_{3} \mathrm{~K}_{\square}}$ and $\mathrm{L}_{G 3 i K D_{\square}}$, respectively. Whether the latter logics are actually equivalent to the diamond-free fragments of the former logics we have not (yet) investigated.

Most other logics in the literature also contain the modal operator diamond or are above S4 and S5, and therefore different from the logics in this paper. However, our logics $\mathrm{L}_{G 3 i K_{\square}}$ and $\mathrm{L}_{G 3 i K D_{\square}}$ occur as the system $\mathbf{H K}_{\square}$ in (Božić and Došen, 1984) and $\mathbf{H D}_{\square}$ in (Došen, 1985), respectively. In these two papers the logics are defined via Hilbert style proof systems and proved to be sound and complete with respect to certain classes of Kripke models with two accessibility relations. In the same way as for the logics in (Simpson, 1994), the equality between $\mathbf{H K}_{\square}$ and $\mathrm{L}_{G 3 i K_{\square}}$, and $\mathbf{H D}_{\square}$ and $\mathrm{L}_{G 3 i K D_{\square}}$ can be seen to hold.

In (Litak, 2014), the logic $\mathrm{K}^{\mathrm{i}}$ is defined in the same manner as the logic Intuitionistic $K$ without $\diamond$ discussed above, except that instead of including all theorems of IPC in the axioms only the axioms of an axiomatization of IPC are included, where any axiomatization might do. As these two logics are obviously equal, $\mathrm{K}^{i}$ is equal to $\mathrm{L}_{G_{3 i} K_{\square}}$ as well. In a similar way it can be shown that the logic NVi from (Litak, 2014) is the same as $\mathrm{L}_{G_{3} i K D_{\square}}$. 
In (Wolter and Zakharyaschev, 1999), the logic $\mathbf{I n t K}_{\square}$ is defined syntactically as well as semantically, and using the syntactic characterization it can easily be established that it is equal to $\mathrm{L}_{\mathrm{G}_{3 i} \mathrm{~K}_{\square}}$.

\subsection{Future work}

In any endeavor that requires a terminating calculus for some intermediate or intuitionistic modal logic, it seems worthwhile to try extend the calculus G4ip to a calculus for the given logic in such a way that termination is preserved. A first step in the extension of our results in (Iemhoff, 2018b) on uniform interpolation to other intuitionistic modal logics, such as $\mathrm{iGL}_{\square}$, would be the development of extensions of G4ip for such logics. Furthermore, it would be interesting to establish whether the intuitionistic modal logics from the literature that contain the diamond operator have a sequent calculus similar to the ones developed in this paper.

Acknowledgements. I thank Marta Bílková for several enjoyable discusssions on uniform interpolation in Utrecht, Leiden, and Helsinki. The help of an anonymous meticulous referee is gratefully acknowledged.

\section{References}

Bílková, M., Interpolation in modal logic, $\mathrm{PhD}$ thesis, Charles University Prague, Czech Republic, 2006.

BíLKovÁ, M., 'Uniform interpolation and propositional quantifiers in modal logics', Studia Logica 85(1):259-271, 2007.

BožIć, M. and K. DošEn, 'Models for normal intuitionistic modal logics', Studia Logica 43(3):217-245, 1984.

Dershowitz, N. and Z. Manna, 'Proving termination with multiset orderings', Communications of the ACM 22:465-476, 1979.

DošEn, K., 'Models for Stronger Normal Intuitionistic Modal Logics', Studia Logica 44(1):39-70, 1985.

Dyckhoff, R., 'Contraction-Free Sequent Calculi for Intuitionistic Logic', Journal of Symbolic Logic 57(3):795-807, 1992.

Gabbay, D.M. and L.L. Maxsimova, Interpolation and definability. Clarendon Press, 2005.

Ghilardi, S. and M. Zawadowski, 'Undefinability of Propositional Quantifiers in the Modal System S4', Studia Logica 55:259-271, 1995.

Ghilardi, S. and M. Zawadowski, Sheaves, Games, and Model Completions: A Categorical Approach to Nonclassical Propositional Logics, Trends in Logic (Book 14), Springer, 2002. 
Hudelmaier, J., 'A Prolog program for intuitionistic propositional logic', SNS-Bericht 88-28, Tübingen, 1988.

Hudelmaier, J., 'Bounds for cut elimination in intuitionistic propositional logic', Archive for Mathematical Logic 31:331-353, 1992.

Hudelmaier, J., 'An $O(n \log n)$-space decision procedure for intuitionistic propositional logic', Journal of Logic and Computation 3:63-76, 1993.

IEMHOFF, R., 'Uniform interpolation and sequent calculi in modal logic', Archive for Mathematical Logic, to appear. Online at https://link.springer. com/article/10.1007/s00153-018-0629-0, 2018a.

IEMHOFF, R., 'Uniform interpolation and the existence of sequent calculi', Logic Group Preprint Series 337, https://lgps.sites.uu.nl/, Utrecht University, 2018b.

Litak, T., 'Constructive modalities with provability smack', in: G. Bezhanishvili, ed., Leo Esakia on duality in modal and intuitionistic logics. Outstanding Contributions to Logic 4, Springer:179-208, 2014.

Maxsimova, L.L., 'Craig's Theorem in superintuitionistic logics and amalgamated varieties of pseudo-boolean algebras', Algebra Logika 16(6):643-681, 1977.

Negri, S., 'Proof Theory for Modal Logic', Philosophy Compass 6(8):523-538, 2011.

PitTs, A., 'On an interpretation of second order quantification in first order intuitionistic propositional logic', Journal of Symbolic Logic 57(1):33-52, 1992.

Shavrukov, V.Y., Subalgebras of diagonalizable algebras of theories containing arithmetic, Dissertationes Mathematicae 323, Instytut Matematyczny Polskiej Akademi Nauk (Warszawa), 1993.

Simpson, A.K., The Proof Theory and Semantics of Intuitionistic Modal Logic. PhDthesis, University of Edinburgh, 1994.

Troelstra, A.S. and H. Schwichtenberg, Basic Proof Theory. Cambridge Tracts in Theoretical Computer Science 43, Cambridge University Press, 1996.

Troelstra, A.S., Corrections to Basic Proof Theory, 1998: http://citeseerx.ist.psu.edu/viewdoc/summary?doi=10.1.1.7.888

VoroB'Ev, N.N., 'The derivability problem in the constructive propositional calculus with strong negation', Doklady Akademii Nauk SSSR 85:689-692, 1952.

Vorob'Ev, N.N., 'A new algorithm for derivability in the constructive propositional calculus', AMS Translations Ser. 2 94:37-71, 1970.

Wolter, F. and M. Zakharyaschev, 'Intuitionistic Modal Logic', in: A. Cantini, E. CASARI, P. MinARI, eds., Logic and Foundations of Mathematics. Synthese Library 280:227-238, 1999. 